\title{
Growth Chambers on the International Space Station for Large Plants
}

\author{
G. D. Massa1a, R. M. Wheeler ${ }^{1}$, R. C. Morrow², and H. G. Levine ${ }^{1}$ \\ ${ }^{1}$ NASA, Kennedy Space Center, FL 32899, USA; 2 ORBITEC, Madison, WI 53717, USA.
}

\section{Abstract}

The International Space Station (ISS) now has platforms for conducting research on horticultural plant species under LED lighting, and those capabilities continue to expand. The 'Veggie' vegetable production system was deployed to the ISS as an applied research platform for food production in space. Veggie is capable of growing a wide array of horticultural crops. It was designed for low power usage, low launch mass and stowage volume, and minimal crew time requirements. The Veggie flight hardware consists of a light cap containing red $(630 \mathrm{~nm})$, blue, $(455 \mathrm{~nm})$ and green $(530 \mathrm{~nm})$ LEDs. Interfacing with the light cap is an extendable bellows/baseplate for enclosing the plant canopy. A second large plant growth chamber, the Advanced Plant Habitat (APH), is will fly to the ISS in 2017. APH will be a fully controllable environment for high-quality plant physiological research. APH will control light (quality, level, and timing), temperature, $\mathrm{CO}_{2}$, relative humidity, and irrigation, while scrubbing any cabin or plant-derived ethylene and other volatile organic compounds. Additional capabilities include sensing of leaf temperature and root zone moisture, root zone temperature, and oxygen concentration. The light cap will have red $(630 \mathrm{~nm})$, blue $(450 \mathrm{~nm})$, green $(525 \mathrm{~nm})$, far red $(730 \mathrm{~nm})$ and broad spectrum white LEDs $(4100 \mathrm{~K})$. There will be several internal cameras (visible and IR) to monitor and record plant growth and operations. Veggie and APH are available for research proposals.

\section{INTRODUCTION}

NASA has two overarching goals for conducting research on crop-type plants in microgravity. The first is to conduct large plant physiology studies to fundamentally understand more about how plants (and associated microorganisms) have evolved with gravity and how they respond to the spaceflight environment and the absence of gravity. While this has extensively been studied in smaller plants, crop-stand level physiology has not been well studied. Facilities for large plants can inform on plant-to-plant interactions and help answer ecological questions on human-plant-microbial ecosystems. These and related questions are important to gain knowledge on the use of plants for bioregenerative life support systems (see below), and may provide insight into ecosystems and agroecosystems on Earth.

The second goal of this research is applied - how can we efficiently grow plants to provide a safe, nutritious and palatable food source for crews in space and in long-duration off-Earth planetary habitation scenarios. At the same time that we are growing plants for food, they can recycle the atmosphere (taking up carbon dioxide and providing oxygen) for astronauts. Plants may also help with waste water processing, and provide pure water through transpiration that can be captured for potable water. Additionally, plants may have a

a E-mail: gioia.massa@nasa.gov 
psychological benefit for astronauts living and working in extreme and hostile environments. This area of research, to use plants to sustain humans, is a component of bioregenerative life support.

NASA has developed science requirements for two hardware systems to fly on the International Space Station (ISS) to help meet these goals. The Veggie hardware is essentially an open system with little internal environmental control. Veggie plants grow in the same atmosphere that the astronaut crew inhabit, with only lights, air movement, water, and slight containment provided by the hardware. This makes Veggie an ideal system to study planthuman-microbe interactions. Veggie was designed with a goal of providing a pick-and-eat diet of salad crops for the crew. It is an open growth platform where a variety of plant growth techniques can be tested. Veggie components are designed to be replaceable, and the lighting control software can also be customized with minor modifications. Veggie is currently in use on the ISS, with a second Veggie unit scheduled to fly by 2017.

The second growth facility is the Advanced Plant Habitat (APH). While Veggie is essentially a bare-bones, customizable plant growth system, the APH will be a highperformance controlled environment chamber with extensive monitoring and control systems. The APH will allow monitoring and control of all of the cardinal factors of plant growth: light, temperature, atmosphere, water and nutrients. In addition, real-time system control, imaging capability from multiple angles, and high data resolution are important aspects of this hardware. The APH was designed so that individual investigators can customize the plant growth environment as required for their investigations. The APH is scheduled to fly in 2017.

Both Veggie and APH are available for plant growth investigations proposed through mechanisms including NASA Research Announcements (NRAs) and the Center for the Advancement of Science in Space (CASIS) organization.

\section{VEGGIE CAPABILITIES}

The Veggie deployable vegetable-production system, developed by the Orbital Technologies Corporation (ORBITEC, Madison, WI), is a modular, low mass, low power, and low crew-time requiring unit with the versatility to grow a variety of crops on the ISS, in planetary habitat environments, or in transit vehicles (Morrow et al, 2005; Morrow and Remiker, 2009). Salad crops recommended for space-life support scenarios that could be grown in Veggie include crops such as lettuce, spinach, chard, mizuna, tomato, pepper, green onion, radish, herbs and strawberry (Wheeler, 2009), however other proposed bioregenerative life support crops such as wheat and soybean could be tested in Veggie, and petri dishes can also be installed for small plant studies. The Veggie unit consists of three primary subsystems: an LED light cap that can be set to different light intensities, a transparent, extendable bellows enclosure, and a reservoir (Fig. 1) that can interface with small plant grow-bags called plant pillows attached via elastic cords. Plant pillows are packages containing growth substrate, controlled release fertilizer, and seeds that are sent to the ISS dry and installed and hydrated on orbit. Plant pillows are designed to be passively watered from a reservoir, however other growing scenarios may utilize the Veggie facility. Veggie usage and plant watering systems are evolving as flight validation tests with different plant types proceed. The expandable bellows provide a moderate level of enclosure from the direct cabin environment and can be used at variable heights between 5 and $45 \mathrm{~cm}$ below the 
light cap. The bellows and baseplate are secured to the light cap via magnetic closures and stabilized with extensible flexible arms. The unit baseplate measures $29.2 \mathrm{~cm}$ by $36.8 \mathrm{~cm}$ providing $0.11 \mathrm{~m}^{2}$ growing area and a total usable volume of up to $0.48 \mathrm{~m}^{3}$. Veggie units may be used sequentially or simultaneously, to provide different cropping scenarios. In preparation for flight, the original Veggie concept was upgraded by ORBITEC for improved capabilities and insertion into the ISS Expedite-the-Processing-of-Experiments-to-the-SpaceStation (ExPRESS) racks, which provide power to the hardware and a separate air cooling loop for the LED light cap with walls to the rear, left sides of the hardware, and another payload on the right side. Walls are white and would likely positively impact the edge radiation within the hardware in this environment. The Veggie plant growth unit's atmospheric conditions (temperature, relative humidity, and $\mathrm{CO}_{2}$ levels) are maintained by the cabin air of the ISS. Veggie bellows are made from a fluorinated polymer. The first Veggie flight unit was installed in the Columbus (European Laboratory) module of the ISS on May 7, 2014 with a hardware validation test using lettuce that ran for 33 days. A second validation test, also with lettuce, was conducted in July and August, 2015. Validation tested the functionality of the hardware for growing an edible crop plant, with data collected on plant growth and food safety.

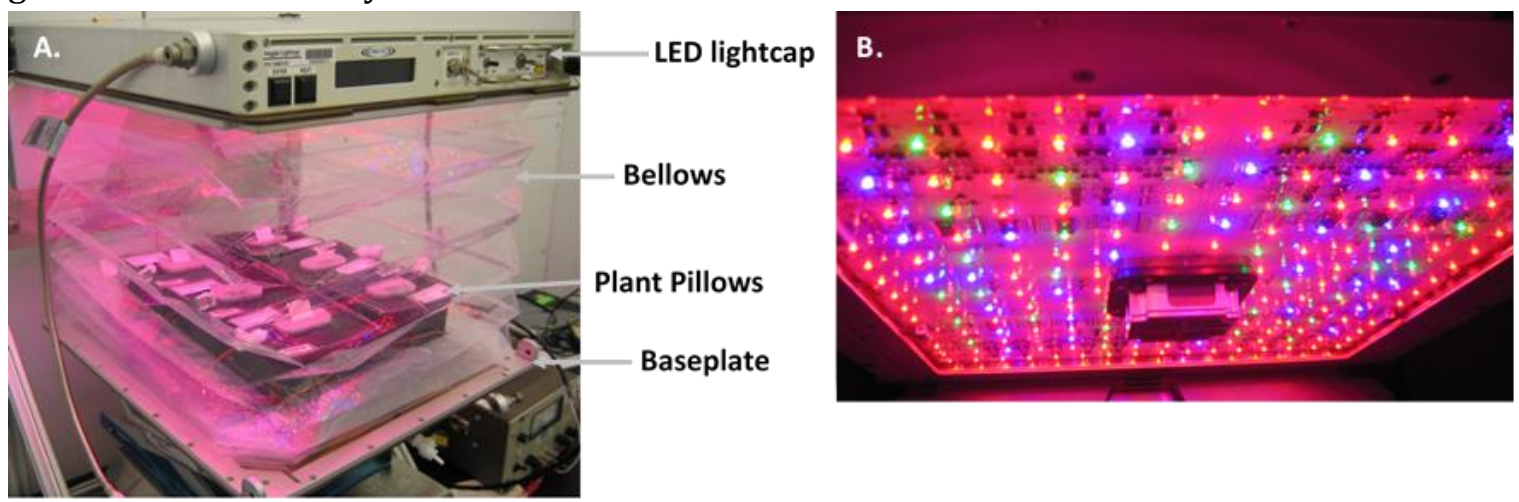

Figure 1. A) The Veggie vegetable production system, B) Close-up view of the Veggie lightcap.

Environmental data within the Veggie chamber can be collected using a temperature and relative humidity data logger placed inside the chamber. Reference environmental data averages over the course of the first Veggie growth period are given in Table 1. These are based on a 16-h day / night cycle with lights on high settings (Table 2), bellows at $32.5 \mathrm{~cm}$ and humidity changing due to lettuce plant growth. The incoming air temperature, humidity and $\mathrm{CO}_{2}$ levels vary with ISS cabin air environments, which are dependent on the number of astronauts present, the set points, and the functional capacity of the environmental control system. The interior Veggie conditions also vary with light and fan settings, bellows height, and the stage of growth of plants in the hardware. 
Table 1. ISS and Veggie environmental data from May-June 2014. Veggie interior environment was measured using an inserted HOBO data logger (U12-011 Onset, Borne, MA). Data points are averages \pm standard deviations. ISS environment data were downlinked from the space station sensors (not immediately adjacent to the hardware). Data are averages for the 33 days of experimentation from germination until harvest, with ISS environment data collected every minute and HOBO data collection at 15 minute intervals. Number of data points for each row is indicated in the first column. Although the day-to-nigh transitions were instantaneous, for calculation simplicity to avoid the photoperiod transition period, night data are averages of the hours between midnight and 05:45 AM and day data are averaged between 10:00 AM and 15:45 PM to give 24 data points for each period.

\begin{tabular}{lcccc}
\hline & $\mathbf{n}$ & Temperature $\left({ }^{\circ} \mathrm{C}\right)$ & Relative Humidity $(\%)$ & $\mathbf{C O}_{2}$ Level $\left(\mu \mathrm{mol} \mathrm{mol}^{-1}\right)$ \\
\cline { 2 - 5 } ISS environment & 49301 & $22.2 \pm 0.2$ & $43.9 \pm 3.7$ & $2798.9 \pm 677.7$ \\
Data Logger-Day & 792 & $24.8 \pm 0.1$ & $38.2 \pm 0.5$ & - \\
Data Logger-Night & 792 & $21.7 \pm 0.1$ & $44.3 \pm 0.7$ & - \\
\hline
\end{tabular}

\section{VEGGIE LIGHTING}

The Veggie flight hardware light cap contains red $(630 \mathrm{~nm})$, blue, $(455 \mathrm{~nm})$ and green $(530 \mathrm{~nm})$ LEDs. Design requirements for these light settings were based on set point measurements at $10 \mathrm{~cm}$ below the light cap at nine points $(n=9)$ evenly distributed over the chamber area with three preset levels of red and blue and one level of green (Table 2). These requirements do not apply to areas within $5 \mathrm{~cm}$ of the bellows walls. The Veggie hardware was able to meet these requirements for intensity, and the uniformity requirements were met for the red light. For the green and blue light at $30 \mu \mathrm{mol} \mathrm{m}^{-2} \mathrm{~s}^{-1}$, these requirements were too stringent so a waiver was given. Variation is due to the relatively small number of blue and green LEDs in the hardware, so light at these low levels was less uniform.

Table 2. Design requirements and potential maximum custom levels for Veggie light cap photosynthetic photon flux (PPF) range and spatial uniformity. All wavelength requirements are $\pm 10 \mathrm{~nm}$ and numbers are presented in $\mu \mathrm{mol} \mathrm{m}^{-2} \mathrm{~s}^{-1} \pm \%$.

\begin{tabular}{lccc}
\hline Setting & $\mathbf{6 3 0} \mathbf{n m ~ r e d}$ & $\mathbf{4 5 5} \mathbf{~ n m}$ blue & $\mathbf{5 3 0} \mathbf{~ n m ~ g r e e n ~}$ \\
\hline Low & $120 \pm 10 \%$ & $30 \pm 10 \%$ & - \\
Medium & $240 \pm 10 \%$ & $60 \pm 10 \%$ & - \\
High & $360 \pm 10 \%$ & $90 \pm 10 \%$ & - \\
On & - & - & $30 \pm 5 \%$ \\
Potential & 550 & 150 & 100 \\
Custom & & & \\
\hline
\end{tabular}

While the base settings of the Veggie lightcap are given here, Veggie has the capability to accept custom light levels through an RS-232 interface. These custom light settings are limited to the values given in Table 2, and may be limited further based on the light cap thermal control system requirements.

Extensive light mapping was conducted within the Veggie hardware and custom light maps are created for new investigations as needed. Basic light maps are illustrated in Figures 2 and 3. A calibrated quantum sensor with meter mounted on a levelling plate was used (LICOR LI-190, LI-COR Biosciences, Lincoln, NE) and a $2.5 \mathrm{~cm}$ grid, $37.5 \mathrm{~cm} \mathrm{x} 30 \mathrm{~cm}$ was assembled to represent the baseplate of the veggie hardware. The grid had 15 points in depth and 12 in width, providing 180 measurement points. This grid was placed on a levelled laboratory jack below the Veggie lightcap with the bellows and baseplate removed. The 
laboratory jack was set to the desired height and light levels were mapped. The quantum sensor was centered at each grid point to take the measurements and 3-dimensional light maps were obtained of different light levels of red, blue and green light. Heights selected were $43.5 \mathrm{~cm}$ (the maximum height of the Veggie bellows with the flexible support arms fully extended) and $32.5 \mathrm{~cm}$ (the height selected for the Veggie validation test as it would provide approximately $250 \mu \mathrm{mol} \mathrm{m}^{-2} \mathrm{~s}^{-1}$ at the height of a Veggie plant pillow). Figure 2 shows the light maps at $43.5 \mathrm{~cm}$ with lights set to high while Figure 3 shows those at $32.5 \mathrm{~cm}$ with lights set to medium.

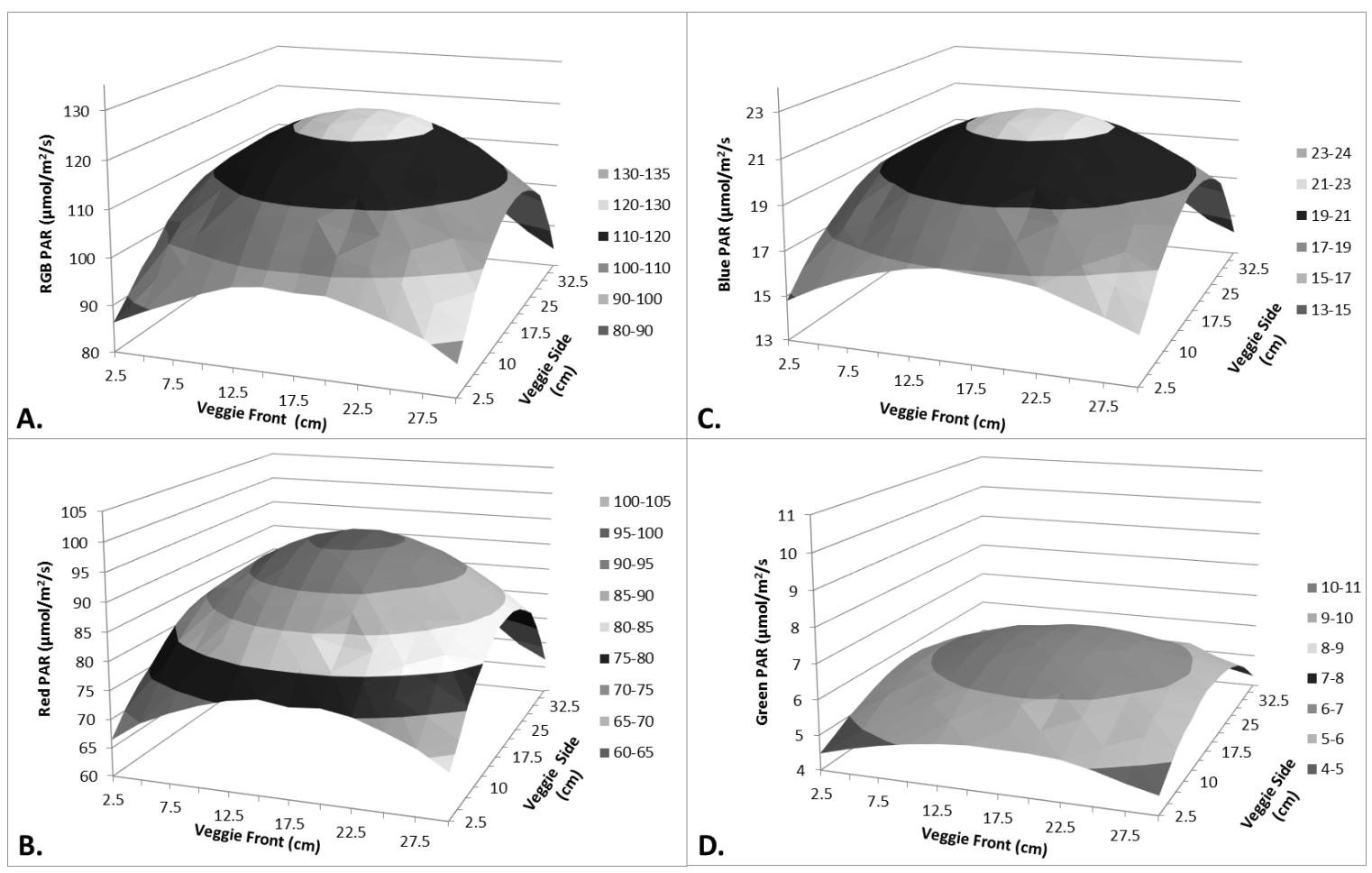

Figure 2. Light maps of the Veggie hardware at $43.5 \mathrm{~cm}$ from the lightcap. Data are A) For red and blue lights on high and green on, B) for red lights only, C) for blue lights only, and D) for green lights only. Data represent 3-dimensional plots of 180 points on the Veggie baseplate.

In addition to detailed mapping without the Veggie bellows, a 12-point light map was obtained within the bellows to assess the bellows effects. Data from these positions without bellows were subtracted from values with bellows to obtain an added effect due to reflection. An average of $5.7 \mu \mathrm{mol} \mathrm{m}^{-2} \mathrm{~s}^{-1}\left( \pm 1.4 \mu \mathrm{mol} \mathrm{m}^{-2} \mathrm{~s}^{-1}\right)$ additional light is added from reflection off the bellows at the $43.5 \mathrm{~cm}$ height with red and blue lights on high and green lights on. When required, external radiation penetrating the transparent Veggie bellows from ISS lighting will be mitigated through the use of opaque bellows, which have been developed for plants requiring absolute darkness during night cycles. The impact of these on light distributions within Veggie has not yet been assessed. 


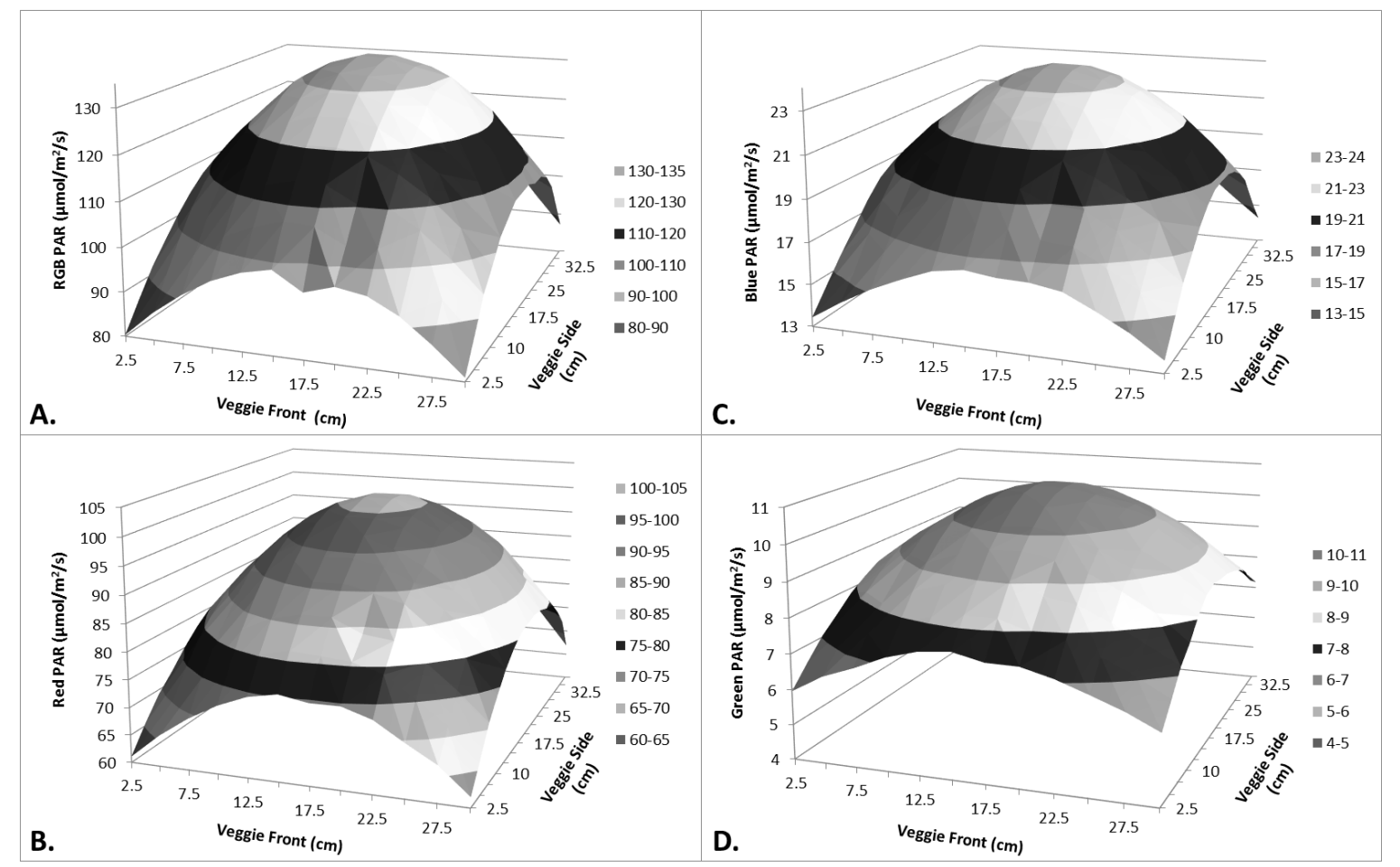

Figure 3. Light maps of the Veggie hardware at $32.5 \mathrm{~cm}$ from the lightcap. Data are A) for red and blue lights on medium and green on, B) for red lights only, C) for blue lights only, and D) for green lights only. Data represent 3-dimensional plots of 180 points on the Veggie baseplate.

\section{ADVANCED PLANT HABITAT CAPABILITIES}

The advanced plant habitat is designed for continuous or multi-generational plant growth studies up to 135 days. Most components of the hardware are designed as orbital replacement units (ORUs). Figure 4 shows the APH Engineering Development (flight precursor) Unit (EDU) hardware. APH will have external viewing windows that can be closed off to prevent light leakage, and a top view color camera, side view color camera, and side view infrared (IR) camera. Figure 5 shows images of wheat from both views in the EDU. The maximum shoot height is $45 \mathrm{~cm}$ with a growth area of $0.19 \mathrm{~m}^{2}$. The science carrier base root tray ( $45.4 \mathrm{~cm} \times 40.8 \mathrm{~cm} \times 5.1 \mathrm{~cm}$ ) consists of a porous tube water delivery system, root zone oxygen sensors (Morrow and Crabb, 2000), temperature sensors, and substrate moisture sensors in four independently controllable root modules (not shown). Initial science carrier concept of operations has the porous tubes providing water to a solid substrate containing controlled release fertilizer, within which the tubes are embedded and root growth will be contained. Other growth scenarios will be dependent on scientific needs of specific investigations. The carrier also has a leaf temperature sensor (Apogee Instruments SI-121: Narrow Field of View Infrared Radiometer Sensor, Logan UT) on a gooseneck arm that can be adjusted to cover any area within the carrier or moved completely out of the growing area to prevent shading. This carrier is removable from the growth chamber and the concept of operations is that the APH growth chamber and associated control systems will be permanently installed in the ISS, while the science carriers will be sent pre-planted from Earth, grown on ISS, and returned to Earth following plant harvest. 

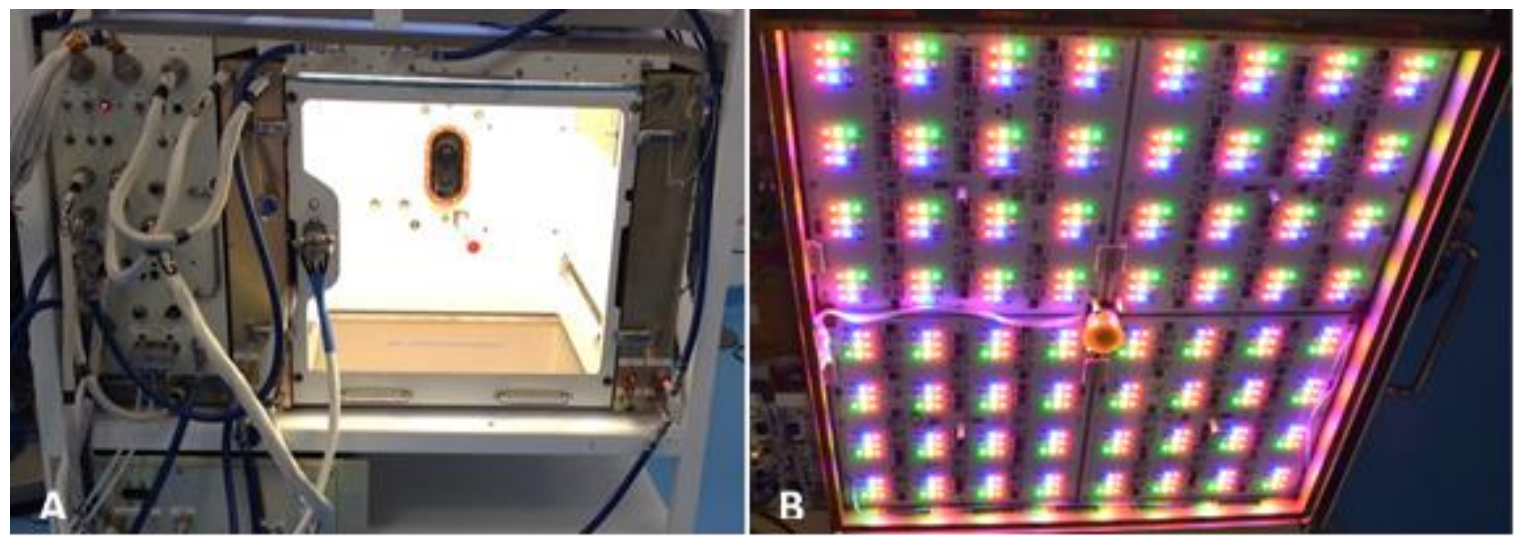

Figure 4. A: The APH EDU B: The APH lightcap.
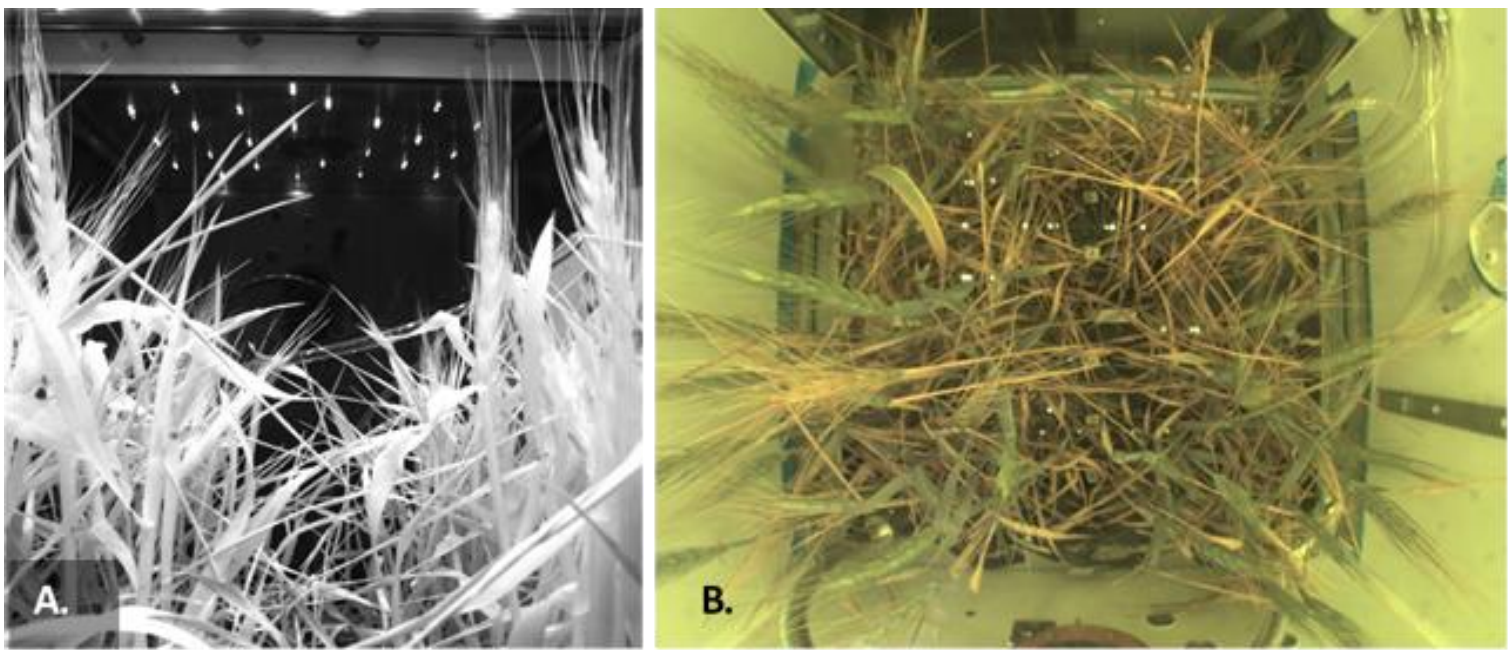

Figure 5. Images from the APH EDU cameras. A: Side IR camera, B: Overhead color camera. An image taken with the side color camera is not shown.

The growth chamber provides precise environmental control. Temperature can be controlled between $18-30^{\circ} \mathrm{C}\left( \pm 1^{\circ} \mathrm{C}\right)$. Relative humidity can be controlled between $50-86 \%$ $( \pm 5 \%)$, and internal condensate from transpiration generated humidity is measured and recycled. The $\mathrm{CO}_{2}$ concentration can be controlled between $500-5000 \mu \mathrm{mol} \mathrm{mol}^{-1}( \pm 50 \mu \mathrm{mol}$ mol- $^{-1}$ ) and APH has the capability to deactivate $\mathrm{CO}_{2}$ control to perform $\mathrm{CO}_{2}$ draw-downs for photosynthesis studies. Oxygen levels are monitored in the shoot zone. Air flow is controllable between $0.3-1.5 \mathrm{~m} \mathrm{~s}^{-1}$ and ethylene is scrubbed to below 25 ppb using a $\mathrm{KMnO}_{4}$ filter. Air in the hardware is filtered, (MERV 13, $\leq 2.6 \mu \mathrm{m}$ particles), and can also be sampled for return to Earth and analysis.

The lighting system in the APH allows 0-1000 $\mu \mathrm{mol} \mathrm{m}^{-2} \mathrm{~s}^{-1} \mathrm{PPF}$ settable in increments of $50 \mu \mathrm{mol} \mathrm{m}^{-2} \mathrm{~s}^{-1}$ (measured at $15 \mathrm{~cm}$ below the light bank) (Fig. $4 \mathrm{~B}$ ). Light wavelengths and ranges are provided in Table 3. Light uniformity at all levels is planned to vary no more than $\pm 15 \%$ over the area of the chamber at greater than $5 \mathrm{~cm}$ from the walls. Light is measured internally by a quantum sensor, (LI-COR 190 SZ, LI-COR Biosciences, Lincoln, NE), and a red/far red sensor, (Skye 110, Skye Instruments, Powys, UK), on a swing-out arm. APH will have manual light blocking to prevent any external light sources from entering the hardware. 
Table 3. Design requirements for the APH photosynthetic photon flux (PPF) range.

\begin{tabular}{lc}
\hline Wavelength & PPF $\left(\mu \mathrm{mol} \mathrm{m} \mathrm{m}^{-2} \mathrm{~s}^{-1}\right)$ \\
\hline Red $(630 \mathrm{~nm})$ & $0-600$ \\
Blue $(450 \pm 10 \mathrm{~nm})$ & $0-400$ \\
Green $(525 \pm 10 \mathrm{~nm})$ & $0-100$ \\
Broad Spectrum White $(4100 \mathrm{~K})$ & $0-600$ \\
Far Red $(730 \mathrm{~nm})$ & $0-50$ \\
No light & $\leq 0.02$
\end{tabular}

Light and dark cycles can be programmed to intervals with a resolution of 1 minute, and in the event power is interrupted the cycle will reestablish at the point in the lighting cycle where it would have been if power had not been interrupted. The light cycle can be overridden manually on ISS or remotely from the ground.

\section{CONCLUSIONS}

The Veggie and APH hardware provide new opportunities for large plant research in microgravity. Veggie allows applied food production research incorporating impacts from humans and human associated microorganisms. In addition to plant growth in response to microgravity and light, studies in Veggie can examine aspects of plant-microbial ecology, human nutrition and dietary augmentation, and human behavioral health. The APH will enable tightly controlled plant physiology studies. APH will allow scientists to unlock the effects of gravity within larger plants, with the ability to study growth, photosynthesis, water use, and other factors in real-time. These units complement a suite of ISS research hardware including microscopes, gloveboxes, freezers and other capabilities to assess or return samples. Veggie and APH are available now for proposals through NASA research announcements, International Life Science research announcements, and CASIS.

\section{ACKNOWLEDGEMENTS}

The authors wish to acknowledge the work of engineering and project management teams at KSC and ORBITEC for providing data on the hardware and help with this manuscript. Light mapping data were taken with the help of Nicole Dufour, Gerard Newsham, LaShelle Spencer, and Debra Dukes. The authors wish to thank NASA's ISS research program for supporting development of the hardware and NASA's Space Life and Physical Sciences Space Biology program for developing science needs and funding verification tests.

\section{Literature Cited}

Morrow, R.C. and Crabb, T.M. (2000). Biomass production system (BPS) plant growth unit. Adv. Space Res. 26, 289-298 http://dx.doi.org/10.1016/S0273-1177(99)00573-6

Morrow, R.C., and Remiker, R.W. (2009). A deployable salad crop production system for lunar habitats. SAE Tech. Paper 2009-01-2382 http://dx.doi.org/10.4271 /2009-01-2382

Morrow, R.C., Remiker, R.W., Mischnick, M.J., Tuominen, L.K., Lee, M.C., and Crabb, T.M. (2005). A low equivalent system mass plant growth unit for space exploration. SAE Tech. Paper 2005-01-2843

http://dx.doi.org/10.4271/2005-01-2843

Wheeler, R.M. (2009). Roadmaps and strategies for crop research for bioregenerative life support systems: a compilation of findings from NASA's advanced life support meetings. NASA Tech. Mem. 2009-214768. 